\title{
Cerebral Differences in Explicit and Implicit Emotional Processing - An fMRI Study
}

\author{
J. Scheuerecker ${ }^{a} \quad$ T. Frodl $^{a} \quad$ N. Koutsouleris ${ }^{a} \quad$ T. Zetzsche $^{a} \quad$ M. Wiesmann ${ }^{b}$ \\ A.M. Kleemann ${ }^{b} \quad$ H. Brückmann ${ }^{b} \quad$ G. Schmitt ${ }^{a} \quad$ H.-J. Möller ${ }^{a} \quad$ E.M. Meisenzahla \\ Departments of a Psychiatry and Psychotherapy, and ${ }^{\mathrm{b}}$ Neuroradiology, Ludwig Maximilians University, \\ Munich, Germany
}

\section{Key Words}

$\mathrm{fMRI} \cdot$ Facial expression - Emotional processing, implicit, explicit

\begin{abstract}
The processing of emotional facial expression is a major part of social communication and understanding. In addition to explicit processing, facial expressions are also processed rapidly and automatically in the absence of explicit awareness. We investigated 12 healthy subjects by presenting them with an implicit and explicit emotional paradigm. The subjects reacted significantly faster in implicit than in explicit trials but did not differ in their error ratio. For the implicit condition increased signals were observed in particular in the thalami, the hippocampi, the frontal inferior gyri and the right middle temporal region. The analysis of the explicit condition showed increased blood-oxygen-level-dependent signals especially in the caudate nucleus, the cingulum and the right prefrontal cortex. The direct comparison of these 2 different processes revealed increased activity for explicit trials in the inferior, superior and middle frontal gyri, the middle cingulum and left parietal regions. Additional
\end{abstract}

J.S. and T.F. contributed equally to the preparation of the manuscript.

\section{KARGER \\ Fax +41 613061234 E-Mail karger@karger.ch}

(c) 2007 S. Karger AG, Basel 0302-282X/07/0561-0032\$23.50/0 www.karger.com signal increases were detected in occipital regions, the cerebellum, and the right angular and lingual gyrus. Our data partially confirm the hypothesis of different neural substrates for the processing of implicit and explicit emotional stimuli.

Copyright $\odot 2007$ S. Karger AG, Basel

\section{Introduction}

The integration of emotional and cognitive processes is reflected by cerebral activation, especially in limbic and prefrontal brain regions $[1,2]$. There are numerous connections between the orbital prefrontal cortex and the amygdala, and also between the ventrolateral and the dorsolateral prefrontal cortex [2]. This neocortical modulation of amygdala responses in functional investigations was observed particularly for fearful stimuli [3].

Earlier functional studies of healthy controls revealed that emotional facial expressions activate the ventral occipital cortex, the middle and superior temporal gyri and the amygdala system $[4,5]$. Furthermore, there is evidence from neuroimaging and brain lesion studies for 2 discrete neural systems for emotional perception, one operating at a conscious level, the other at a nonconscious level [6-9]. These latter investigations were either performed using masked emotional stimuli or included
Thomas Frodl

Department of Psychiatry and Psychotherapy, Ludwig Maximilians University Nussbaumstrasse 7, DE-80336 Munich (Germany)

Tel. +49895160 3370, Fax +498951605343

E-Mail thomas.frodl@med.uni-muenchen.de 
blindsight subjects, thus the emotional stimuli were indeed processed without consciousness. The applied implicit paradigm of the present study is not nonconsciousness but without explicit focus in emotional facial expression. Comparing different studies it is important to differentiate exactly between the used paradigm and their nomenclature. E.g. the study of Hariri et al. [10] suggests that in their perceptual matching task, which is equivalent to the explicit condition of the current investigation, 'subjects tend to match the faces based on perceptual characteristics ... but not need to judge or interpret the emotion'. In contrast, during their so-called labeling condition, subjects had to judge which adjective (sad, angry, ...) best matches the presented face. To avoid confusion we will use the term 'implicit' for a nonemotional focus but consciously visible stimuli, the term 'explicit' for emotion matching and the term 'unconscious' for paradigms with masked stimuli.

Implicit processing of emotional faces (judgment of other facial cues, e.g. gender) leads to increased involvement of the amygdala or the hippocampus [4, 11]. Matching faces by expression results in an activation of the cingulum, the medial temporal lobe and the ventral prefrontal cortex [12] and the amygdala [10]. There are different assumptions about the role of different kinds of emotional stimulus (happy vs. sad vs. angry, etc.). Some investigations propose a nonselective response, in particular in the amygdala, across different emotional stimuli, when subjects have to focus on emotional facial expression [13], other data confirm distinct signal changes for different facial expressions during tasks similar to our implicit condition [14].

We aim to test the hypothesis that explicit and implicit processing of facial expressions underlies distinct neural substrates. Implicit processing is suggested to operate subcortically, and explicit processes include cortical areas that are more directly accessible through conscious attention.

A second aim of the study was to create a paradigm to be used for further studies with depressive patients. Therefore, we decided not to use fearful but angry and sad emotional faces, assuming that especially the processing of sad stimuli is a relevant aspect for the generation and symptomatology of depressive disorders. In contrast to previous studies, the current investigation combined a gender judgment task - similar to the works of Critchley et al. [4], Gur et al. [11] and Blair et al. [14] - and an expression judgment task. In contrast to Hariri et al. [10], we chose a paradigm excluding verbalizing of emotional expressions as applied in the 'labeling task' of this previous investigation. Instead, we used 2 tasks with equivalent stimuli (only faces, no words or the like), firsttime gender matching and second-time expression matching were required. To our knowledge this is the first investigation which tries to compare different modi of facial processing with regard to emotional components by requiring solely visual facial perception.

\section{Methods}

\section{Participants}

Twelve subjects (5 males and 7 females), recruited from the city of Munich, volunteered. All participants were right-handed according to the modified version of the Edinburgh Handedness Inventory [15]. In addition to the usual exclusion criteria for functional magnetic resonance imaging (fMRI) investigations, subjects were excluded if aged under 18 or over 65 , pregnant, suffering from psychiatric, neurological or other severe medical illness, or if they had a history of alcohol/drug abuse. The mean age of the subjects was 41.75 years $(\mathrm{SD}=11.56)$, with a mean of 12.50 years of education $(\mathrm{SD}=1.17)$. As this sample was a subgroup from a different investigation comparing healthy subjects and patients with major depression [Frodl et al., 2007, in preparation], it was screened by an experienced psychiatrist.

Every participant signed informed consent forms. The protocol complied with the Declaration of Helsinki and was approved by the Ethics Commission of the Ludwig Maximilians University of Munich.

\section{Stimuli}

Stimuli consisted of faces drawn from the Facial Expression of Emotion: Stimuli and Tests [16]. There were 48 triplets of emotional faces (sad or angry), arranged in a block design, resulting in 8 blocks of 6 triplets each, interspersed with 9 control blocks. Control blocks consisted of 6 triplets each, presenting simple geometrical, black figures (squares, triangles, circles, ellipses). For the explicit trial each triplet contained either 3 female or 3 male faces. The participants were instructed to choose which faces belonged together with regard to their emotional expression.

For the implicit trial each triplet contained 1 male or female face as the target and 2 other faces of mixed gender. Participants were asked to determine the gender that matched the target face. The target faces alternately showed angry and sad emotions. Responses were given with an fMRI-compatible LumiTouch system using 2 keys for choosing the right or left face in the lower line of the triplet. Each triplet was presented for $5.3 \mathrm{~s}$, resulting in a total length of about 9 min for each trial (8 blocks with emotional faces, 9 control blocks with geometrical figures). The order of trials (explicit, implicit) and of target stimuli was randomized.

\section{Image Acquisition}

Functional images were acquired on a 1.5-tesla Siemens scanner (Siemens Erlangen), using a T2*-weighted gradient echoplanar imaging sequence, sensitive to blood-oxygen-level-dependent contrast $\left(\mathrm{TR}=3,200 \mathrm{~s}, \mathrm{TE}=60 \mathrm{~ms}\right.$, flip angle of $90^{\circ}$, matrix $=64$ $\times 64, \mathrm{FOV}=256 \times 256 \mathrm{~mm}$ ). Two functional runs of $175 \mathrm{con}-$ tiguous volumes were acquired. The volumes comprised 26 axial 
slices of $4 \mathrm{~mm}$ thickness. The first 5 volumes of each run were discarded to reach signal equilibrium. The transaxial functional images covered the whole brain and were positioned parallel to the intercommissural line (AC-PC). Additionally, for each participant a 3-dimensional magnetization prepared rapid gradient echo water excitation (MPrage) was acquired for anatomical localization $(\mathrm{TR}=11.4 \mathrm{~ms}, \mathrm{TE}=4.4 \mathrm{~ms}$, matrix $=256 \times 256 \times 128$, FOV $=256 \times 256 \mathrm{~mm}$, slice thickness $=1.5 \mathrm{~mm})$, comprising 160 slices.

\section{Behavioral Data Analysis}

Reaction times and number of errors for faces were calculated separately for the implicit and explicit condition. A 2-sided Pearson correlation test was used for the variables task quality (error ratio) and quantity (reaction times), as well as for quantity/quality and years of education. For the comparison of the performance for the different conditions a paired $t$ test was used. All tests had a significance level of $p<0.05$.

\section{fMRI Data Analysis}

For data analysis, Statistical Parametric Mapping (SPM2) was used with the following preprocessing steps: realigning all volumes to the sixth scan to correct for subject motion (exclusion criteria: more than $3 \mathrm{~mm}$ ), coregistration of the functional and structural data sets, spatial normalizing [by using sinc interpolation method $(9 \times 9 \times 9)]$ into a standard stereotactic space, using a template of the Montreal Neurological Institute and smoothing the data with an 8-mm Gaussian kernel. Statistical parametric maps were calculated based on a voxel-by-voxel method, using a general linear model [17]

First-Level Analysis

In the first-level analysis the statistical design matrix included 2 sessions (implicit and explicit) with 2 regressors and 1 constant, respectively.

Each regressor consisted of a box-car function convolved with an estimated hemodynamic response function. Thus the expected hemodynamic response to the experimental stimulus was modeled using the relative contributions of a delayed box-car reference wave function as well as confounding variables (wholebrain activity and low-frequency variations).

After parameter estimation, contrast images were constructed for explicit triplets $>$ control stimuli, implicit triplets $>$ control stimuli, implicit triplets $>$ explicit triplets and explicit triplets $>$ implicit triplets.

Furthermore, we performed an additional analysis to identify possible habituation effects by comparing the first 4 emotional blocks and the second 4 blocks for both conditions (early emotional blocks $>$ late emotional blocks and early emotional blocks $<$ late emotional blocks).

\section{Second-Level Analysis}

Each subject's contrast images entered the second-level analysis, using 1-sample t-tests for intragroup analysis. Resulting SPM $\mathrm{t}$-maps were thresholded with $\mathrm{p}<0.05$ (false discovery rate corrected). An extent threshold of 10 voxels was applied.

The anatomic localization of significant voxels was identified using the SPM toolbox Automated Anatomic Labeling, which is described by Tzourio-Mazoyer et al. [18].
Table 1. Behavioral data

\begin{tabular}{lll}
\hline & $\mathrm{t}_{\text {face }}$ & Correct answers \\
\hline Implicit & 1,280 & $88.5 \%$ \\
Explicit & 2,785 & $87.6 \%$ \\
p value & 0.000 & 0.693 \\
\hline
\end{tabular}

\section{Results}

\section{Behavioral Data}

Analysis of behavioral data revealed significantly longer reaction times for explicit trials but no differences in the number of correct answers between the 2 conditions (see table 1). Due to technical problems complete behavioral data sets could not be recorded for 4 subjects.

We were also interested in the correlation of task quality (correct answers) and task quantity (reaction time). A significant negative correlation between quality and quantity was detected for the explicit condition $(\mathrm{r}=-0.74$, $\mathrm{p}=0.036)$ but not for the implicit trial $(\mathrm{r}=-0.30, \mathrm{p}=$ 0.939). No significant correlations were found for task quality/quantity and years of education.

\section{fMRI Data}

\section{Explicit Processing}

The volunteers showed bilateral signal increases in the cerebellum, the caudate nucleus and the cingulum. Unilateral signal increases were observed in the right inferior frontal gyrus and the left middle frontal gyrus (see table 2 b, fig. 1 ).

The largest clusters (more than 1,000 voxels) were found in the right cerebellum (30,267 voxels; extended into both fusiform and lingual gyri, left superior, middle and inferior occipital gyrus), the right inferior frontal gyrus (5,542 voxels; extended into right middle frontal gyrus, right insula and precentral gyrus) and the left middle frontal gyrus (8,285 voxels, extended into left superior, inferior and middle frontal gyrus, left precentral gyrus, left insula and right middle cingulum).

\section{Implicit Processing}

Increased bilateral activities were observed in the hippocampus, the thalamus and the inferior frontal gyrus. Unilateral signal increases were detected in the left lingual gyrus, the right superior occipital gyrus, the right middle temporal gyrus, the right precentral gyrus and the the left inferior parietal lobe (see table $2 a$, fig. 1). 
Table 2. fMRI data

a Implicit $>$ control

\begin{tabular}{lrrrrr}
\hline Anatomical region & X & Y & Z & $\begin{array}{l}\text { Cluster } \\
\text { size }\end{array}$ & $\begin{array}{l}\text { T } \\
\text { value }\end{array}$ \\
\hline L lingual gyrus & -30 & -82 & -16 & 7,926 & 9.73 \\
R superior occipital gyrus & 24 & -64 & 34 & 1,212 & 7.15 \\
R thalamus & 22 & -12 & 0 & 63 & 5.11 \\
& 8 & -18 & -2 & 14 & 3.18 \\
L thalamus & -24 & -30 & 2 & 34 & 4.01 \\
R hippocampus & 12 & -18 & -14 & 24 & 4.93 \\
& 18 & -28 & -8 & 95 & 4.32 \\
L hippocampus & -30 & -20 & -12 & 21 & 4.07 \\
R inferior frontal gyrus & 40 & 40 & 14 & 154 & 4.53 \\
& 34 & -2 & 26 & 25 & 3.91 \\
L inferior gyrus & -38 & 28 & -8 & 65 & 3.95 \\
& -38 & 10 & 26 & 382 & 5.02 \\
R middle temporal gyrus & 58 & -46 & -2 & 28 & 4.97 \\
L inferior parietal lobule & -34 & -56 & 42 & 27 & 4.02 \\
R precentral gyrus & 48 & 4 & 32 & 85 & 4.25 \\
\hline
\end{tabular}

b Explicit $>$ control

\begin{tabular}{lrrrrr}
\hline R cerebellum & 34 & -68 & -24 & 30,267 & 14.63 \\
& 16 & -42 & -20 & 11 & 2.71 \\
L cerebellum & -20 & -34 & -24 & 26 & 3.81 \\
R caudate nucleus & 20 & -18 & 22 & 35 & 3.47 \\
& 2 & 14 & 14 & 12 & 3.30 \\
L caudate nucleus & -18 & -12 & 18 & 11 & 2.70 \\
R middle cingulum & 20 & -14 & 42 & 13 & 2.83 \\
L anterior cingulum & -8 & 4 & 28 & 54 & 3.78 \\
R inferior frontal gyrus & 40 & 36 & 12 & 5,542 & 10.52 \\
L middle frontal gyrus & -26 & 10 & 54 & 8,285 & 10.11 \\
\hline
\end{tabular}

c Explicit $>$ implicit

\begin{tabular}{lrrrrr}
\hline R middle occipital gyrus & 40 & -84 & 10 & 171 & 12.36 \\
& 28 & -74 & 28 & 42 & 4.42 \\
L middle occipital gyrus & -42 & -86 & 8 & 168 & 5.37 \\
& -32 & -76 & 26 & 84 & 4.86 \\
R inferior occipital gyrus & 40 & -68 & -12 & 692 & 7.82 \\
R cerebellum & 30 & -68 & -42 & 24 & 4.01 \\
L cerebellum & -38 & -66 & -22 & 406 & 8.44 \\
& -30 & -70 & -44 & 265 & 6.13 \\
& -8 & -78 & -34 & 624 & 5.89 \\
Vermis & 2 & -60 & -40 & 243 & 5.08 \\
L inferior parietal lobule & -36 & -46 & 54 & 10 & 4.34 \\
L superior parietal lobule & -30 & -60 & 52 & 119 & 5.38 \\
R angular gyrus & 30 & -58 & 42 & 345 & 5.82 \\
R inferior temporal gyrus & 60 & -62 & -4 & 38 & 5.63 \\
L middle temporal gyrus & -62 & -56 & 6 & 51 & 4.53 \\
R lingual gyrus & 34 & -88 & -20 & 29 & 4.73 \\
R middle cingulum & 6 & 26 & 38 & 102 & 4.53 \\
R inferior frontal gyrus & 50 & 34 & 16 & 1,629 & 8.63 \\
L inferior frontal gyrus & -38 & 30 & 14 & 1,221 & 7.02 \\
R middle frontal gyrus & 34 & 54 & 4 & 11 & 4.55 \\
L superior frontal gyrus & 0 & 42 & 50 & 101 & 5.33 \\
\hline
\end{tabular}

Cerebral Differences in Explicit and Implicit Emotional Processing

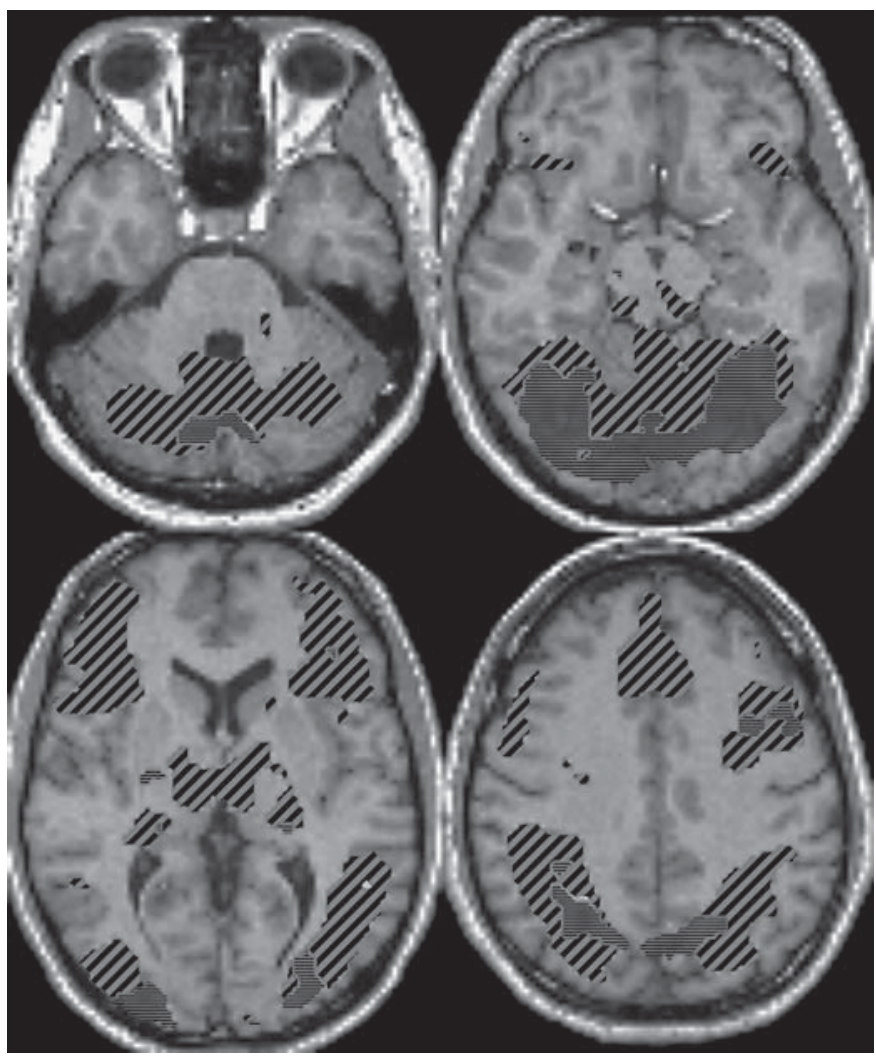

Fig. 1. Increased signals in explicit (horizontal stripes, background) and implicit (diagonal stripes, foreground) processing for $\mathrm{p}<0.05$ corrected (false discovery rate).

The largest clusters (more than 1,000 voxels) were detected in the left lingual gyrus (7,926 voxels, extended into both fusiform gyri, cerebellum and lingual gyrus, right lingual gyrus, right inferior and left middle and inferior occipital gyrus) and the right superior occipital gyrus (1,212 voxels; extended into the precuneus bilaterally, the right middle and left superior occipital gyrus, right angular gyrus).

\section{Explicit vs. Implicit Processing}

No enhanced activity was found in the direct comparison of implicit and explicit trials (implicit $>$ explicit). The other way round (explicit $>$ implicit), participants showed increased bilateral activity in the cerebellum, the inferior frontal gyrus, the occipital lobe and the temporal lobe. Unilateral signal increases were observed for the right gyrus angularis, the left parietal lobe, the right middle cingulum, the right lingual gyrus, the left superior frontal and the right middle frontal gyrus (see table $2 \mathrm{c}$, fig. 2). 


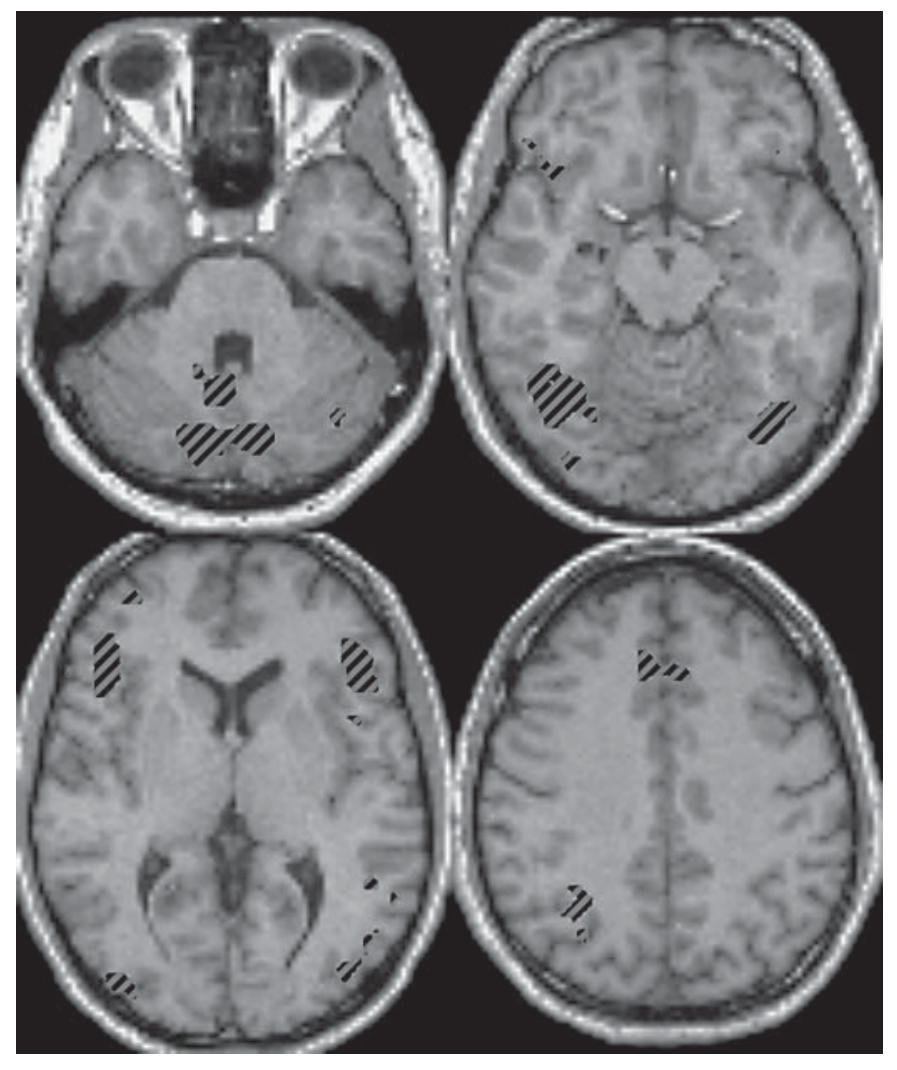

Fig. 2. Increased signal in explicit compared to implicit processing (explicit $>$ implicit) for $\mathrm{p}<0.05$ corrected (false discovery rate).

The largest clusters (more than 1,000 voxels) were observed for explicit $>$ implicit in the right inferior frontal gyrus (1,629 voxels; extended into right precentral gyrus and right insula) and the left inferior frontal gyrus $(1,221$ voxels; extended into the left insula, and the left precentral gyrus).

\section{Habituation Analysis}

No differences for early and late blocks were observed, neither for the explicit nor for the implicit condition.

\section{Discussion}

\section{Implicit vs. Explicit Processing}

The first aim of our study was to compare implicit and explicit processes. We found evidence for similar neural substrates, the explicit task recruiting additional networks. Thus our results partially confirm the hypothesis of different neural substrates for the processing of implicit and explicit emotional stimuli.
Our data particularly concurred with the study of Critchley et al. [4], in which significantly greater activity in the middle temporal gyrus was observed for explicit than for implicit trials. The middle temporal gyrus seems to be crucial for the processing of facial expressions and movements [19]. Thus it seems evident that the matching of gender evoked a lower level of activity than the emotion-matching task because the identification of facial expressions is more decisive for the latter task. Nevertheless, we have to consider that the explicit trial of Chritchley et al. requires a semantic judgment which resembles the labeling task used by Hariri et al. [10], so the data are not directly comparable.

Our most convincing result was the increased activity in the frontal cortex. The inferior frontal gyrus was involved in implicit as well as in explicit processing of emotional expressions, whereby the involvement was significantly greater for the latter. This increased activity was also present in the superior (left hemisphere) and the middle (right hemisphere) frontal gyrus. These very large clusters for explicit $>$ implicit were not observed in the study of Critchley et al. Our participants had to match faces, while those in the study of Critchley et al. had to judge 1 face by semantic labeling (happy or neutral). Therefore, it seems that the different cognitive demand and the higher emotional load of our paradigm require distinct cognitive strategies.

Furthermore, we detected a significant difference in implicit and explicit processing in the right middle cingulum. This is a subregion of the anterior cingulum, which plays an important role in (focused) attention [20] and whose activation increases with task difficulty [21, 22]. The cingulum is also proposed to be involved in the processing of emotional information, but with regard to the subdivisions of the anterior cingulate cortex described by Bush et al. [23] our findings seem to reflect additional cognitive effort rather than emotional processing.

Indeed, our results indicate that stronger activation and extended clusters in the same regions for the explicit as for the implicit task support the interpretation that the emotional matching required a more effortful comparing of parts of the faces, while gender matching is more holistic and effortless (see fig. 1).

Consequently, on the one hand, we suggest that this signal increase is required due to the higher cognitive demands and task difficulty of the explicit task. On the other hand, we hypothesize that this signal increase is also the consequence of a deeper and more salient processing during explicit conditions. The subjects reacted signifi- 
cantly more slowly in explicit than in implicit tasks. This extra time for processing seems to compensate for the higher task difficulty in explicit trials, regarding the equivalent error ratios.

A recent study [24] that applied a passive emotional task was able to confirm a complex cerebral network, as proposed by Haxby et al. [5]. In this model, the inferior occipital gyrus, fusiform gyrus and the superior temporal sulcus form the 'core' system, and the amygdala, hippocampus, inferior frontal gyrus and orbitofrontal cortex build an 'extended' system which processes facial expression. Comparing explicit and implicit processing we found significant differences in activation in some of these structures. Interestingly these clusters are also the largest ones with cluster sizes of 692 voxels in the left inferior occipital gyrus, 1,629 voxels in the right inferior frontal gyrus and 1,221 voxels in the left inferior frontal gyrus. Thus it appears that different kinds of process (implicit vs. explicit) have different effects on the core units as well as the extended units of the face perception system. Numerous studies have shown cerebellar involvement in cognitive and emotional functions [25-27]. We could observe cerebellar signals for explicit but not for implicit trials. The notion that the cerebellum is involved in emotional processing has only recently gained popularity, and further studies are needed to clarify the coordinative role for this region, which is still understudied. Furthermore, the data analysis revealed stronger activation in the left inferior and superior parietal lobule for explicit than for implicit processing. Thus our data suggest that explicit processing of facial expression is rather under top-down control, including frontoparietal networks. On the contrary, implicit processing is suggested to be based on bottom-up processes. However, as discussed in the next section, we failed to detect stronger activations of subcortical regions as amygdala and hippocampus for explicit versus implicit processing, which would support more pronounced bottom-up processes in implicit emotional processes than in explicit processing. With regard to this model we assume that including a larger sample size to get more statistical power, the observed hippocampal activity for implicit $>$ control would endure the direct comparison of the contrast implicit $>$ explicit.

\section{Amygdala and Prefrontal Cortex}

The most surprising result, which was in contrast to most of the previous investigations $[3,4,11,13,28,29]$, was the lack of enhanced activation in the amygdala. The important work of Hariri et al. [3] was based on fearful stimuli, using the International Affective Picture System, and is thus not directly comparable with our results. Bleich-Cohen et al. [28] were interested in the effect of differential stimuli also evoking fear. They compared differential effects of fearful scenes, faces and sentences and found greater activity in the amygdala with scenes than with faces or sentences. However, the recent work of Fitzgerald et al. [13] investigated amygdala activity in response to multiple expressions of facial emotions (fearful, disgusted, angry, sad, neutral, happy). The main result was an overall increase of amygdala activity. They concluded that the amygdala has a general-purpose function in the processing of facial information. This would confute the assumption that our failure to detect amygdala activity is the consequence of mixing different kinds of emotional expression.

Another fMRI investigation by Hariri et al. [10] used a block design similar to the one used in our study, but using faces with expressions of fear and anger. Nevertheless, they reported a consistent amygdala activity for emotional processing, which cannot be confirmed by our results. A previous study by Blair et al. [14] investigated the different patterns of activity for angry and sad faces. In contrast to Fitzgerald et al. [13], they reported a signal increase in the amygdala and temporal gyrus for sad faces and signal increases in the orbitofrontal cortex for angry faces. Using mixed triplets we observed (for explicit processing) signal increases in the right inferior and the left middle frontal gyrus as well as in the left anterior cingulum. This result could be the consequence of a greater sensibility for angry faces than for sad faces. Unfortunately, we did not interview the participants about their impression and their way of solving the emotional tasks. This information could have given insight into 2 important questions: (1) Could they recognize and name the emotions correctly? (2) Did they match the faces by focusing on the facial expressions themselves or by identifying typical physiological features (e.g. a wrinkled forehead) without focusing on the whole facial expressions? Our results rather support the latter (more cognitive) method.

An older fMRI investigation by Sprengelmeyer et al. [30] - which also revealed a lack of amygdala activity emphasized the importance of the inferior frontal gyrus. They proposed separate neural pathways for different facial emotional expressions. These pathways have a common end point of projection in the inferior frontal gyrus. As Posamentier and Abdi [31] reason in their review about processing faces and facial expressions, the inferior gyrus becomes active for different facial expressions and 
plays a critical role in the integration of information in facial expressions.

Further explanations for the lack of amygdala involvement could be methodological aspects such as habituation effects due to the applied block design, too high a TE to detect signal changes in subcortical regions or a single subject's artifacts in the amygdala region. However, as the analysis of habituation effects revealed, rapid amygdala activation was not found. Also not enough artifacts could be detected to render this explanation plausible (for this issue see also [32]). Additionally, no relevant artifacts of the amygdala region could be detected by using the SPM toolbox ArtifactRepair (as presented by Mazaika et al. at the Human Brain Mapping (HBM), 2005; http://gablab. stanford.edu/tools.htm).
In summary, our results emphasize the role of the frontal cortex as a general unit for the processing of emotional facial expressions, especially when the task includes comparing and identifying emotional faces instead of simply focusing on emotional stimuli. The hypothesis of different neural substrates for explicit and implicit processing can only partially be confirmed by our results. However, we suggest that the observed hippocampal activations (for implicit $>$ control) would endure the direct statistical comparison (implicit > explicit) investigating a larger sample size.

\section{Acknowledgments}

We would like to thank B. Burgermeister for the technical support. This study was supported by the Lilly Foundation.

\section{References}

$\checkmark 1$ Barbas H: Connections underlying the synthesis of cognition, memory, and emotion in primate prefrontal cortices. Brain Res Bull 2000;52:319-330.

$\checkmark 2$ Mcdonald AJ, Mascagni F, Guo L: Projections of the medial and lateral prefrontal cortices to the amygdala: a Phaseolus vulgaris leucoagglutinin study in the rat. Neuroscience 1996;71:55-75.

$\checkmark 3$ Hariri AR, Mattay VS, Tessitore A, Fera F, Weinberger DR: Neocortical modulation of the amygdala response to fearful stimuli. Biol Psychiatry 2003;53:494-501.

4 Critchley H, Daly E, Phillips M, Brammer M, Bullmore E, Williams S, Amelsvoort TV, Robertson D, David A, Murphy D: Explicit and implicit neural mechanisms for processing of social information from facial expressions: a functional magnetic resonance imaging study. Hum Brain Mapp 2000;9:93105.

5 Haxby JV, Hoffman EA, Gobbini MI: The distributed human neural system for face perception. Trends Cogn Sci 2000;4:223233.

6 De Gelder B, Vroomen J, Pourtois G, Weiskrantz L: Non-conscious recognition of affect in the absence of striate cortex. Neuroreport 1999;10:3795-3763.

7 Driver J, Vuilleumier P, Eimer M, Rees G: Functional magnetic resonance imaging and evoked potential correlates of conscious and unconscious vision in parietal extinction patients. Neuroimage 2001;14:68-75.

8 Morris JS, Ohman A, Dolan JR: Conscious and unconscious emotional learning in the human amygdala. Nature 1998;393:467470 . $\checkmark 9$ Whalen PJ, Rauch SL, Etcoff NL, McInerney SC, Lee MB, Jenike MA: Masked presentations of emotional facial expressions modulate amygdala activity without explicit knowledge. J Neurosci 1998;18:411-418.

10 Hariri AR, Bookheimer SY, Mazziotta JC: Modulating emotional responses: effects of a neocortical network on the limbic system. Neuroreport 2000;11:43-48.

11 Gur RC, Schroeder L, Turner T, McGrath C, Chan RM, Turetsky BI, Alsop D, Maldjian J, Gur RE: Brain activation during facial emotion processing. Neuroimage 2002;16:651662.

12 George MS, Ketter TA, Gill DS, Haxby JV, Ungerleider LG, Herscovitch P, Post RM: Brain regions involved in recognizing facial emotion or identity: an oxygen-15 PET study. J Neuropsychiatry Clin Neurosci 1993;5: 384-394.

13 Fitzgerald DA, Angstadt M, Jelsone LM, Nathan PJ, Phan KL: Beyond threat: amygdala reactivity across multiple expressions of facial affect. Neuroimage 2006;30:1441-1448.

14 Blair RJ, Morris JS, Frith CD, Perrett DI, Dolan RJ: Dissociable neural responses to facial expressions of sadness and anger. Brain 1999; 122:883-893.

15 Oldfield RC: The assessment and analysis of handedness: the Edinburgh inventory. Neuropsychologia 1971:9:97-113

16 Young A, Perrett D, Calder A, Sprengelmeyer R, Ekman P: Facial Expressions of Emotion: Stimuli and Tests (FEEST). Bury St Edmunds, Thames Valley Test Company, 2000.
7 Friston K, Holmes A, Worsley K, Polina J, Frith C, Frackowiak R: Statistical parametric maps in functional imaging: a general linear approach. Hum Brain Mapp 1995;2:189210.

18 Tzourio-Mazoyer N, Landeau B, Papathanassiou D, Crivello F, Etard O, Delcroix N, Mazoyer B, Joliot M: Automated anatomical labeling of activations in SPM using a macroscopic anatomical parcellation of the MNI MRI single-subject brain. Neuroimage 2002; 15:273-289.

19 Puce A, Allison T, Bentin S, Gore JC, McCarthy G: Temporal cortex activation in humans viewing eye and mouth movements. J Neurosci 1998;18:2188-2199.

20 Gevins A, Smith ME, McEvoy L, Yu D: Highresolution EEG mapping of cortical activation related to working memory: effects of task difficulty, type of processing, and practice. Cereb Cortex 1997;7:374-385.

21 Ishihara T, Yoshi N: Multivariate analytic study of EEG and mental activity in juvenile delinquents. Electroencephalogr Clin Neurophysiol 1972;33:71-80.

22 Paus T, Koski L, Caramanos Z, Westbury C: Regional differences in the effects of task difficulty and motor output on blood flow response in the human anterior cingulate cortex: a review of 107 PET activation studies. Neuroreport 1998;9:37-47.

23 Bush G, Luu P, Posner MI: Cognitive and emotional influences in anterior cingulate cortex. Trends Cogn Sci 2000;4:215-222.

24 Ishai A, Schmidt CF, Boesiger P: Face perception is mediated by a distributed cortical network. Brain Res Bull 2005;67:87-93. 
25 Andreasen NC, Nopoulos P, O’Leary DS, Miller DD, Wassin T, Flaum M: Defining the phenotype of schizophrenia: cognitive dysmetria and its neural mechanisms. Biol Psychiatry 1999;46:908-920.

26 Schmahmann JD: Disorders of the cerebellum: ataxia, dysmetria of thought, and the cerebellar cognitive affective syndrome. J Neuropsychiatry Clin Neurosci 2004;16: 367-378.
7 Wiser AK, Andreasen NC, O'Leary DS, Watkins GL, Boles Ponto L, Hichwas RD: Dysfunctional cortico-cerebellar circuits cause cognitive dysmetria in schizophrenia. Neuroreport 1998;9:1895-1899.

28 Bleich-Cohen M, Mintz M, Pianka P, Andelman F, Rotshtein P, Hendler T: Differential stimuli and task effects in the amygdala and sensory areas. Neuroreport 2006;17:13911395.

$\checkmark 29$ Chen C-H, Lennox B, Jacob R, Calder A, Lupson V, Bisbrown-Chippendale R, Suckling J, Bullmore E: Explicit and implicit facial affect recognition in manic and depressed states of bipolar disorder: a functional magnetic resonance imaging study. Biol Psychiatry 2006;59:31-39.
0 Sprengelmeyer R, Rausch M, Eysel UT, Przuntek H: Neural structures associated with recognition of facial expressions of basic emotions. Proc Biol Sci 1998;265:19271931.

31 Posamentier MT, Abdi H: Processing faces and facial expressions. Neuropsychol Rev 2003;13:113-143.

32 Merboldt AD, Fransson P, Bruhn H, Frahm $\mathrm{J}$ : Functional MRI and the human amygdala? Neuroimage 2001;14:253-257. 